Conclusions SEAM enables hidden costs associated with dysfunction to be re-allocated to activities with much higher professional added value. It is an attractive approach to monitor the time needed to transform our low-quality clinical pharmacy services into a competitive environment of modern and reactive Pharmaceutical Care services.

No conflict of interest.

\section{GRP-009 ADHERENCE AND NUMBER OF TABLETS IN ANTIRRETROVIRAL TREATMENT}

\section{doi:10.1136/ejhpharm-2013-000276.009}

F Gutiérrez Nicolás, MM Viña Romero, MG Callejon Callejon, P Diaz Ruiz, FJ Merino Alonso, JA Martin Conde. Hospital Ntra. Sra. de Candelaria, Pharmacy, Santa Cruz de Tenerife, Spain

Background Antiretroviral efficacy is closely related to the degree of adherence

Purpose To determine the adherence to highly active antiretroviral therapy (HAART) in HIV-infected patients with once-daily dosing regimens, depending on the number of tablets.

Materials and Methods Two-month observational study (MayJune 2010 of selected patients on HAART who collected their medicines in the pharmacy with the following inclusion criteria: adult patients on HAART for more than a year, who were not included in any clinical trials, mentally competent and who obtained the medicines exclusively in our LEU.

The SMAQ survey was used to assess adherence. Adherence data, along with the number of tablets and demographic characteristics of the patients were tabulated and analysed using Excel.

Results 223 patients were included in the study. 39.5\% $(n=88)$ had once-daily regimens. 72 were men and 16 women. The mean age was 44.3 years and 7.35 years on HAART. The mean adherence was $67.05 \%$.

The study population was divided into two groups: one tablet (OT) $(\mathrm{n}=49)$ and two or more tablets (MT) $(\mathrm{n}=39)$. Baseline characteristics were homogeneous in the two groups. However adherence rates were $71.42 \%$ vs. $61.54 \%$ respectively $(p=0.3268)$. Conclusions Simple dosing regimens facilitate adherence to HAART. In our study we found that OT patients were more adherent that MT patients. Although the difference in adherence was not statistically significant, we believe that this difference may have high clinical impact on controlling the disease.

No conflict of interest.

\section{GRP-010 ADHERENCE TO ORAL CANCER TREATMENT: THE ROLE OF THE HOSPITAL PHARMACIST IN THERAPEUTIC SUCCESS}

\section{doi:10.1136/ejhpharm-2013-000276.010}

F Santoleri, P Sorice, R Lasala, RC Rizzo, A Costantini. Santo Spirito General Hospital of Pescara, Hospital Pharmacy, Pescara, Italy

Background The management of cancer treatment has changed considerably in recent years with the entry into the market of novel oral cancer agents. Although treatment at home improves patient compliance, in practise it's difficult to assess the quality of treatment without the supervision of healthcare professionals.

Purpose To monitor patients at home and to assess the variables influencing adherence to treatment. We sought to educate, discuss and establish effective communication with patients in order to minimise the barriers between patients and physicians.

Materials and Methods From July 2012, hospital pharmacists have provided their haematology-oncology patients with a selfreport medicines diary. Patients were asked to write the date, time, treatment dosage and concomitant treatments, as well as to describe their health status and report any side effects. Data were saved in a database created for the purpose. Treatment adherence was calculated as Medicines Possession Ratio according to the treatment indications in the patient diary.

Results From July 2012 to October 2012, a total of 261 patients were asked to participate in the study and to fill out a self-reported diary. 243 patients agreed to participate in the study, of these 86 completed and returned the self-report diaries (41\%) to the hospital pharmacy. The percentage of adherence to treatment was significantly higher in those patients who completed the medicines diaries compared to those who did not use the medicines diary $(0.99$ vs. 0.88$)$. The reported side effects indicated that medicines were well tolerated and did not cause discontinuation of treatment.

Conclusions The preliminary data of this patient-oriented research emphasises the importance of promoting dialogue in order to optimise home treatment. The hospital pharmacist plays a key role in promoting and improving adherence to treatment by analysing side effects and concomitant treatment and, in addition, by reinforcing patients' awareness of the importance of following the prescription schedule correctly.

No conflict of interest.

\section{GRP-011 ADHERENCE TO TYROSINE KINASE INHIBITOR THERAPY IN CHRONIC MYELOID LEUKAEMIA}

doi:10.1136/ejhpharm-2013-000276.011

'V González Rosa, ${ }^{2} \mathrm{MM}$ Viña Romero, ${ }^{3} \mathrm{~F}$ Gutiérrez Nicolás, 'R Gavira Moreno, 'MT Moreno Carvajal, 'F Gómez de Rueda, 'R Gázquez Pérez, 'P Gómez Germá, 'A Almendral Vicente, 'MT Gómez de Travecedo y Calvo. 'Hospital SAS Jerez de la Frontera, UGC-Farmacia, Jerez de la Frontera (Cádiz), Spain; ${ }^{2}$ Hospital Universitario Nuestra Señora de Candelaria, UGC-Farmacia, Tenerife, Spain; ${ }^{3}$ Hospital Universitario de Canarias, UGC-Farmacia, Tenerife, Spain

Background Improved survival associated with tyrosine kinase inhibitor (TKI) treatment has transformed chronic myeloid leukaemia (CML) into a long-term disease, but therapeutic success is challenged with poor medicines adherence. Controlling side effects in combination with patient education that includes direct communication between the pharmacist and the patient are essential components for maximising the benefits of TKI treatment.

Purpose To estimate the adherence to oral chemotherapy and describe side effects with TKI treatment and their impact on adherence in patients with CML.

Materials and Methods An 18-month retrospective observational study (from January 2011 to June 2012) was made on patients diagnosed with CML in which patients were selected who collected medicines in the pharmacy and who were being treated with selected TKIs (imatinib, dasatinib, nilotinib).

The SMAQ interview was used to determinate adherence. Adherence data, side effects and demographic characteristics of the patients were tabulated using Excel. The $\mathrm{x}^{2}$ test was used for categorical variables and the t-test was used for normally-distributed continuous variables using SPSS statistical software.

Results 25 patients were included in the study. 16 were men and 9 were women. The mean age was 60 years (25-88). Imatinib was the first line treatment for all patients. The average adherence was $62.5 \%$

Adherence for patients younger than 50 years was $83.3 \%$ and in older patients was $55.6 \%(\mathrm{P}=0.125)$. Relating to years of treatment: less than 4 years $70.0 \%$ but for longer treatment $57.1 \%$ $(p=0.521)$. Patients with side effects showed less adherence: gastrointestinal disorders ( $80.0 \%$ vs. $64.28 \%, p=0.402)$, musculoskeletal pain (70.0\% vs. $42.8 \% \mathrm{p}=0.188)$.

Conclusions Data suggest that more than one-third of patients are poorly adherent to TKI treatment. Identifying risk factors such as side effects, and educating patients on the need to take 